\title{
1 A semi-empirical model relating flow properties to particle contacts in fine
}

\section{2 binary powder mixtures}

4 Takehiro Kojima and James A. Elliott ${ }^{*}$

5

6 Department of Materials Science and Metallurgy, University of Cambridge,

727 Charles Babbage Road, Cambridge, CB3 0FS, UK

8

$9 \quad *$ Corresponding author. Tel.: +441223 335987; fax: +44 1223334567 .

10 E-mail address: jae1001@cam.ac.uk

14 Keywords: Binary powder mixtures; Bulk cohesion; Internal friction; Coordination number;

15 Bulk density; Incipient shear zone 
1

2

3

4

5

6 composed of toner - resin particles with a diameter of 5-10 $\mu \mathrm{m}$ functioning as a colouring

7 materials - and carrier particles, usually made of magnetic materials with a mean diameter of

$8 \quad 50-100 \mu \mathrm{m}$, which promote the electrostatic charging of the toner particles and carry the

9 toners onto the latent images. Inside the EP printing device, a mixture of toner and carrier

10 particles is intermittently circulated under applied shear and normal stresses in the range of

$11-10 \mathrm{kPa}$. Since the flow behaviour of the developer affects the image quality, it is crucial to

12 control their flow properties appropriately $[2,3]$.

13 Whilst many of the factors affecting the flow properties of two-component developers,

14 such as particle size and shape distributions, composition, mechanical characteristics, etc., do

15 not change significantly during normal operation of EP printing devices, the mixing ratio, or

16 compositional balance, of the constituent particles is one of the important variables to control

17 in the process [1]. Therefore, understanding the relationship between the flow properties and

18 mixing ratio of binary powder mixtures is of great importance for proper control of the flow 
In this study, we present a semi-empirical model which describes the relationship between

2 the bulk flow properties of large-and-small dry fine binary powder mixtures and the ratio of

3 the component powders, incorporating the contacts between the constituent particles.

4 Specifically, the model estimates the number of particle contacts involved in the incipient

5 flow motion, taking into account the change in the width of the incipient shear zone, and links

6 these to changes in the flow properties. In fact, an understanding of the fundamental physics

7 connecting changes in the particle contact network and width of the incipient shear zone to

8 the onset of incipient flow is of wide benefit to a range of granular systems beyond EP

9 printing devices such as the processes of powder materials in pharmaceutical and food

10 industrials.

11 However, despite its importance, the number of fundamental studies exploring the bulk

12 flow properties of large-and-small binary powder mixtures in terms of the mixing ratio of the

13 constituent particles is still limited. There exists some experimental research investigating the

14 relationship between the bulk flow properties and mixing ratio of the binary powder mixtures

15 [4-6], but the analyses of the mechanisms were rather qualitative. Computational modelling

16 studies also exist (e.g. Ref $[7,8])$ but the results are again rather limited due to the high

17 complexity of such systems, although this might potentially be a useful approach with further

18 improvements of computational speed and modelling techniques in the near future. The

19 current study aims to address the gap between the experimental measurements and numerical 
1 simulations towards the ultimate goal of an improved understanding of their linkage.

2 In this study, we firstly investigated the bulk flow properties of fine binary powder 3 mixtures experimentally. We used fine powders to construct model systems of

4 two-component developers used in EP printing devices, thus eliminating any confounding

5 factors such as the varying geometric shape of the constituent powder particles and unknown

6 surface treatments. In a previous study, we successfully performed systematic measurements

7 by using commercially-available toner powders as the smaller constituent of the mixtures [2].

8 However, in present work, in order to develop a model which correctly describes the adhesive

9 property between the constituent particles, we utilised spherical polymer powders as the

10 smaller constituents whose bulk cohesion were systematically controlled.

11 Then, a semi-empirical model, which explains the bulk flow properties of the binary

12 powder mixtures in terms of the contacts of the constituent particles, was developed by

13 estimating the number of particle contacts involved at the point of incipient flow. There exist

14 some studies discussing the particle packing of two- or multi-component powder mixtures

15 from the microscopic viewpoint, relating the bulk packing density to the number of particle

16 contacts (i.e. coordination number), including experimental measurements [9,10], analytical

17 modelling [11-16], and computational simulations [17]. In this study, a geometrical model

18 proposed by Suzuki et al. [12-14], in which the coordination number of the constituent

19 particles in the binary powder mixtures is calculated from the bulk density (or voidage) of the 
1 systems, was used for the estimation of the numbers of particle contacts in the system. The

2 experimental data were then analysed further using the proposed model to determine its

3 validity.

4 The remainder of this article is structured as follows: after describing the experimental

5 methods and materials in Section 2, the results of the measurement of the powder packing

6 structures and flow properties such as bulk cohesion and internal friction of the binary

7 powder mixtures are presented in Sections 3 and 4, respectively. A model equation to relate

8 the particle contacts in the powder mixtures and their flow properties is then introduced in

9 Section 5, and further analyses based on the model are given in Section 6. We conclude by

10 summarising the achievements in Section 7.

13 2. Experimental methods and materials

15 Several types of smaller powders with different bulk cohesions were prepared for the

16 purpose of developing a model describing the adhesive property between the constituent

17 particles properly. Binary powder mixtures were then made using each small powder together

18 with a larger powder, in which the mixing ratio was systematically changed, and the incipient

19 flow properties of the powder mixtures were measured under the same experimental 
1 conditions. The details of the measurement methods of the flow properties, as well as the

2 powder materials used for the binary mixtures, are described in the remainder of this section.

\subsection{Measurement of flow properties}

6 The incipient flow properties of the powder samples were evaluated using a ring shear

7 tester (RST) RST-XS (Dietmer Schulze, Germany). The fundamental principles of shear

8 testing, as well as a description of the RST equipment, are summarised elsewhere in various

9 textbooks and papers (e.g. Refs. $[18,19])$.

10 The series of measurements were conducted under the same experimental conditions as

11 Ref [2]. The powder sample was at first placed into the ring-shaped shear cell of height 13

$12 \mathrm{~mm}$, inner and outer diameters of 32 and $64 \mathrm{~mm}$, respectively, and the lid with radial vanes of

13 height $3 \mathrm{~mm}$ distributed every $22.5^{\circ}$ on its lower surface, was loaded onto the sample. In each

14 measurement, the incipient shear stresses, $\tau_{\mathrm{sh}}$, were measured under normal stresses of $\sigma_{\mathrm{sh}}=$

$150.6,1.1$ and $1.6 \mathrm{kPa}$ after the pre-shear consolidation with a normal stress of $\sigma_{\text {pre }}=2.0 \mathrm{kPa}$.

16 In all cases, the obtained data showed good linearity (correlation coefficients, $R^{2}$, of the

17 linear fitting were greater than 0.994). Based on this, two parameters representing major

18 incipient flow properties, bulk cohesion and internal friction, were obtained from the yield

19 locus, a line fitted to the three measured $\left(\sigma_{\mathrm{sh}}, \tau_{\mathrm{sh}}\right)$ points in the $\sigma-\tau$ plane (i.e. a 
1 Mohr-Coulomb yield locus) expressed by the following equation:

2

$3 \tau=\sigma \cdot \tan \varphi_{\text {lin }}+\tau_{\mathrm{c}}$.

5 In Eq. (1), $\tau_{\mathrm{c}}$, the $\tau$-intercept of a yield locus, represents the bulk cohesive property of the

6 powder sample and is called 'bulk cohesion'. Also, the angle of the slope of the yield locus

$7 \varphi_{\text {lin }}$ is a measure of the internal friction of the powder sample and is termed the "linearised

8 friction angle (LFA)'.

9 The bulk structure of every powder sample was also evaluated in the form of voidage, $\varepsilon$,

10 which represents the volume ratio of the voids in the total volume of powder system. The

11 value of $\varepsilon$ was calculated from the bulk density of the powder, $\rho_{b}$, obtained in the RST

12 measurement, particle densities of the large and small components: $\rho_{\mathrm{p}, 1}$ and $\rho_{\mathrm{p}, \mathrm{s}}$, and the

13 volume ratio of the small powder, $x_{\mathrm{s}}$, by using the following relationship:

14

15

$\varepsilon=1-\frac{\rho_{\mathrm{b}}}{\rho_{\mathrm{p}, 1}\left(1-x_{\mathrm{s}}\right)+\rho_{\mathrm{p}, 1} x_{\mathrm{s}}}$.

17 Each measurement was carried out under standard conditions of $21 \pm 2^{\circ} \mathrm{C}$ and $38-55 \%$

18 relative humidity $(\mathrm{RH})$. The fluctuations of room temperature and $\mathrm{RH}$ did not affect the 19 results. 


\subsection{Powder materials}

Glass ballotini (Ballotini solid soda glass balls, diameter 40-70 $\mu \mathrm{m}$, Sigmund Lindner,

5 Germany) of the same type as in a previous study [2] were used as the larger component in

6 the binary mixtures. Before being used for the flow measurement, the ballotini powder was

7 washed using liquid detergent and rinsed thoroughly in distilled water in order to eliminate

8 fine impurities on the particle surface. After being dried in the cabinet with an internal

9 temperature of approximately $80{ }^{\circ} \mathrm{C}$ for at least 8 hours, it was then sieved using a set of three

10 nested sieves with different mesh sizes (38, 90 and $100 \mu \mathrm{m}$ from bottom) in order to obtain a

11 sufficiently uniform and narrow size distribution. Only the powder remaining on the $38 \mu \mathrm{m}$

12 sieve was used for the evaluation of the flow properties. For the smaller component of the

13 binary mixtures, a polymer powder composed of poly(styrene-co-divinylbenzene) (PS-DVB)

14 microspheres (Sigma-Aldrich, USA) was used without pre-treatment process.

15 The cumulative size distributions of the powders obtained by image analysis are shown in

16 Fig. 1, together with representative SEM images of each type of particles (SEM: JEOL 6340F

17 FEG-SEM). Under the SEM, the constituent particles of both glass ballotini and PS-DVB

18 powders appeared almost spherical, and also the surface of the glass ballotini was confirmed

19 to be almost smooth and impurity-free. Their size parameters such as volume mean diameter, 
$1 d_{50}$, and geometric standard deviation, $\sigma_{\mathrm{g}}$, of each powder were obtained by fitting a

2 log-normal function, which closely followed the cumulative size distribution of each powder.

3 The particle density of each powder was measured by a gas pycnometer (Accupyc 1330,

4 Micromeritics, USA). The values of size parameters and particle density are summarised in

5 Table 1.

6 The flow properties and voidage of the glass ballotini and untreated PS-DVB powders

7 were measured using the RST prior to the evaluations of the binary mixtures. They are listed

8 in Table 2.

9

10 2.3. Surface modification of PS-DVB powder

For the control of the cohesive property of the PS-DVB powder, a dry coating technique,

13 in which nanometre-sized materials are added to the surface of host particles as 'flow

14 regulators' or 'surface additives', was adopted. As discussed in a previous study, the cohesive

15 property of the PS-DVB powders can be systematically controlled over a wide range by

16 changing the type and quantity of silica nanoparticles applied as surface additives. Such

17 surface additives have only a small effect on the internal friction [20]. In this study, $12 \mathrm{~nm}$

18 hydrophobic silica nanoparticles, Aerosil ${ }^{\circledR}$ R805 (Evonik Industries, Germany), were chosen

19 as the surface additives for the PS-DVB powder particles. The BET surface area of the silica 
1 nanoparticles provided by the supplier was $150 \pm 25 \mathrm{~m}^{2} \mathrm{~g}^{-1}$.

2 Four types of surface-modified PS-DVB powders covered with different quantities of 12

3 nm hydrophobic silica nanoparticles: 1, 5, 10, and 100\% theoretical surface coverage (TSC)

4 were prepared based on the results in Ref. [20], in which the bulk cohesion of the PS-DVB

5 powders decreased with increase of the quantity of the silica nanoparticles in the expected

6 range of 90-380 $\mathrm{Pa}$. The TSC was calculated from the surface area of PS-DVB microspheres

7 and total projected area of the silica nanoparticles on the surface of the PS-DVB particles

8 when all of the silica nanoparticles are considered to be existing as primary particles using

9 the mean diameters of their primary particles. For ease of reference, the surface-modified

10 PS-DVB powders are termed as $\operatorname{PS}-\operatorname{DVB}(\chi)$ where $\chi$ represents the TSC of the $12 \mathrm{~nm}$

11 hydrophobic silica nanoparticles as a percentage (i.e. 1, 5, 10 or 100) For instance,

12 PS-DVB(10) denotes a PS-DVB powder covered with 10\% TSC of silica.

13 In order to coat the PS-DVB powders with surface additives, the same process was

14 employed as described in Ref. [23]. A commercially available mixer, composed of a plastic

15 container with a capacity of $400 \mathrm{ml}$ (diameter $80 \mathrm{~mm}$ ) and two stainless steel blades (almost

16 isosceles triangles, with $15 \mathrm{~mm}$ base and $36 \mathrm{~mm}$ height, rotating at approximately 18000

$17 \mathrm{rpm}$ ), was used for the addition of the silica nanoparticles to the surface of the PS-DVB

18 particles. In each case, $30 \mathrm{~g}$ of the PS-DVB powder and an appropriate quantity of silica

19 nanoparticles to achieve the desired TSC were mixed together for 120 s. Fig. 2 shows the 
1 magnified SEM images of the particle surface of each surface-modified PS-DVB powder,

2 which shows that the silica nanoparticles are uniformly covering the PS-DVB particles in

3 each case. In each case, the surface-modified PS-DVB powder was stored for at least $8 \mathrm{~h}$

4 before being mixed with glass ballotini in order to eliminate the possible effect of

5 electrostatic charge due to friction between particles or due to the wall of the bottle during the

6 mixing process.

7 The incipient flow properties and voidage of these surface-modified PS-DVB powders

8 were measured prior to the evaluation of binary powder mixtures as summarised in Table 3 .

9 The bulk cohesion of the surface-modified PS-DVB powder was systematically decreased by

10 up to approximately $270 \mathrm{~Pa}$ by increasing the quantity of the silica nanoparticles applied to

11 the surface of the PS-DVB particles from 1 to $100 \%$ TSC. The values of the LFA were not

12 significantly affected by changing the quantity of silica nanoparticles applied; they were

13 identical to within 5.0\%. These observations correspond to the facts reported in Ref. [20] that

14 increasing the TSC of surface additives systematically decreases the bulk cohesion of the host

15 particles with a small effect on LFA. A linear correlation was found between the bulk

16 cohesions of the surface-modified PS-DVB powders and their voidage, as shown in Fig. 3;

17 the less cohesive they are, the more densely the powders can be compacted. 
2 Binary powder mixtures composed of glass ballotini and surface-modified PS-DVB

3 powders were prepared in varying compositions for the evaluation of their flow properties.

4 The powder mixtures were mixed in a $200 \mathrm{ml}$ plastic bottle by shaking it approximately 250

5 times per minute for 2 minutes with amplitude of approximately $20 \mathrm{~cm}$, as in Ref. [2]. Each

6 mixture was confirmed to be sufficiently well mixed without apparent segregation from the

7 fact that the colour of the sample was uniform throughout. Each mixture was stored for an

8 extended period (at least $12 \mathrm{~h}$ ) before being tested with RST in order to eliminate the possible

9 effects of electrostatic charging.

\section{Packing structure of binary powder mixtures}

The voidage, $\varepsilon$, of the binary mixtures composed of the glass ballotini and

15 surface-modified PS-DVB powders are shown in Fig. 4 as a function of the volume ratio of

16 the PS-DVB powder, $x_{\mathrm{s}}$ (on a logarithmic scale). A common trend was observed for all the

17 data: the voidage shows a 'funnel shape' curve, obtaining a minimum value at approximately

$18 x_{\mathrm{s}}=0.4$ in each case. As seen from Fig 4 , the powder mixtures were compacted more densely

19 by using less cohesive PS-DVB powders for the same $x_{\mathrm{s}}$, over the entire range of $x_{\mathrm{s}}$. 
There exist a number of numerical models for binary powder mixtures illustrating the

2 relationship between the packing density and ratio of the component powders, as summarised

3 elsewhere (e.g. Refs [2,21,22]). Among them, the model proposed by Westman [23] describes

4 the relationship in a relatively simple quadratic equation expressed as follows:

5

$6\left(\frac{V-V_{1} x_{1}}{V_{\mathrm{s}}}\right)^{2}+2 G\left(\frac{V-V_{1} x_{1}}{V_{\mathrm{s}}}\right)\left(\frac{V-x_{1}-V_{\mathrm{s}} x_{\mathrm{s}}}{V_{1}-1}\right)+\left(\frac{V-x_{1}-V_{\mathrm{s}} x_{\mathrm{s}}}{V_{1}-1}\right)^{2}=1$,

8 where $V$ is the specific volume of the powder mixture (equivalent to the reciprocal of $1-\varepsilon$ ),

$9 V_{1}$ and $V_{\mathrm{s}}$ are the partial specific volumes of large and small particles corresponding to the

10 specific volumes of the large and small powders alone, and $x_{1}$ and $x_{\mathrm{s}}$ are the volume ratios of

11 the large and small powders, respectively $\left(x_{1}=1-x_{\mathrm{s}}\right)$. In the original Westman's model [23]

12 or its modified versions [22,24], which show good agreement with the experimental results

13 for non-cohesive powder mixtures, the parameter $G$ is a function of size ratio of the two

14 component powder particles. For fine cohesive powders, $G$ can be regarded as an index

15 representing how easily the binary mixture can be compacted, as mentioned in a previous

16 study [2]. Since the measured values of the voidage in the small $x_{\mathrm{s}}$ region, in each case,

17 shows a more rapid decrease with increase of the PS-DVB powder than the model predicts,

18 Eq. (3) was fitted to the measured voidage for $x_{\mathrm{s}} \geq 0.4$ by using $G$ as a variable parameter.

19 The solid lines in Fig. 4 are the results of these fittings, and the values of $G$ for each line are 
1 listed in Table 4. These values are used in Section 6 to discuss the relationship of the packing

2 structure with adhesive properties of the constituent particles.

As seen in Fig. 4, the measured voidage shows some significant deviation from the values

4 predicted by Eq. (3) in the direction of smaller $\varepsilon$ for the low $x_{\mathrm{s}}$ region $\left(x_{\mathrm{s}}<0.1-0.2\right)$.

5 According to the model, the smaller particles are expected to fill in the voids between the

6 larger particles with little disruption of the bulk packing structure of the larger particles in the

7 low $x_{\mathrm{s}}$ region when the size ratio of large and small constituents, $r=d_{1} / d_{\mathrm{s}}$, is sufficiently

8 large, typically nearly 10 or larger [22-24]. The value of $r$ is 9.17 in this case, which seems to

9 almost meet this condition. Based on these facts, the deviation of the measured voidage from

10 the model observed in small $x_{\mathrm{s}}$ region seems to indicate that the PS-DVB particles added to

11 the glass ballotini powder disrupt the bulk structure of the pure glass ballotini and allow the

12 formation of a denser packing structure. Fig. 4 shows that the PS-DVB particles with a larger

13 quantity of $12 \mathrm{~nm}$ hydrophobic silica nanoparticles disrupt the bulk structure of the glass

14 ballotini to a greater extent, forming denser packing structures according to the magnitude of

15 discrepancies between the calculated (lines) and experimental (squares) values.

\section{4. Flow properties of binary powder mixtures}


In a previous study [2], the bulk cohesion of glass ballotini was reduced by adding minute amounts of toner particles $\left(x_{\mathrm{s}}=0.002-0.005\right)$, which was attributed to the fact that the toner particles disrupted direct contacts between the glass ballotini particles, thus reducing the van

5 der Waals attractive force between them. However, in this study, the bulk cohesion in general

6 increased from that of the pure glass ballotini on the addition of a small quantity of

7 surface-modified PS-DVB particles, as seen in Fig 5(a), which shows the bulk cohesion, $\tau_{\mathrm{c}}$,

8 of the binary powder mixtures of glass ballotini and surface-modified PS-DVB as a function

9 of the volume ratio of the PS-DVB powder. This seems to suggest that the effect of the

10 contacts between glass ballotini and surface-modified PS-DVB particles should also be taken

11 into account. As more PS-DVB particles were added, the bulk cohesion decreased until $x_{\mathrm{s}}$

12 reached approximately 0.05 . Once $x_{\mathrm{s}}$ exceeded $0.07-0.1$, the bulk cohesion of the powder

13 mixtures increased rapidly with increasing the PS-DVB particles. The values of the bulk

14 cohesion converged on that of the surface-modified PS-DVB powders after reaching a peak at

$15 \quad x_{\mathrm{s}}=0.4-0.8$.

16 Furthermore, the bulk cohesion of the powder mixtures containing a less cohesive

17 PS-DVB powder became smaller at the same $x_{\mathrm{s}}$ over the entire range, as seen in Fig 5(a). In

18 fact, when the bulk cohesion is compared with the voidage, shown in Fig. 4, these parameters

19 seem to have a correlation with each other; the denser the mixture, the higher the bulk 
1 cohesion, which seems to suggest a strong effect of the bulk flow properties of the powder

2 mixtures on the packing structure of the particles. Their relationship is discussed in more

3 detail in Section 6.

4

5

6

7

8 mixtures $\varphi_{\text {lin }}$ and volume ratio of the smaller component, $x_{\mathrm{s}}$, is shown in Fig. 5(b). In general,

9 the dependence of the LFA on $x_{\mathrm{s}}$ displayed a funnel-shape curve in each case, having a

10 minimum at $x_{\mathrm{s}}=0.1-0.3$, depending on the quantity of the silica nanoparticles added to the

11 surface of the PS-DVB particles. In each case, the LFA of the powder mixtures was almost

12 constant, or showed slight decrease at $x_{\mathrm{s}}<0.05-0.07$, and then decreased rapidly to the

13 minimum point with increasing $x_{\mathrm{s}}$. In more detail, the LFA of the mixture composed of the

14 surface-modified PS-DVB powder with a smaller quantity of silica nanoparticles (i.e. more

15 cohesive PS-DVB powder) started decreasing rapidly at smaller values of $x_{\mathrm{s}}$. The values of $x_{\mathrm{s}}$

16 where the LFA reached the minimum, $x_{\mathrm{s}}\left(\varphi_{\operatorname{lin}, \min }\right)$, also showed a systematic trend; the more

17 cohesive the surface-modified PS-DVB, the smaller the value of $x_{\mathrm{s}}\left(\varphi_{\operatorname{lin}, \min }\right)$. Furthermore, the

18 minimum values of the LFA, $\varphi_{\text {lin,min, }}$ became smaller as a less cohesive surface-modified

19 PS-DVB was used in a mixture. This seems to reflect that the more $12 \mathrm{~nm}$ hydrophobic silica 
1 nanoparticles cover the surface of PS-DVB particles, the lower the friction between the

2 surface-modified PS-DBV particles and glass ballotini becomes. Once the LFA reached a

3 minimum at $x_{\mathrm{s}}\left(\varphi_{\text {lin,min }}\right)$, it increased with further increase of $x_{\mathrm{s}}$ and converged on the LFA of

4 the surface-modified PS-DVB powders in each case. Further discussion in terms of the

5 relationship with particle contacts is given in Section 6.

6

7

\section{5. Model relating flow properties and particle contacts}

9

In this section, we develop a semi-empirical model to relate one of the flow properties of

11 the binary powder mixtures, bulk cohesion $\tau_{\mathrm{c}}$, to the packing structure in terms of the average

12 number of particle contacts. In order to estimate the numbers of particle contacts in the

13 powder mixtures, a model equation was applied based on the geometric analysis proposed by

14 Suzuki et al. [12-14], which provides the average coordination number of the constituent

15 particles of binary powder mixtures from their bulk structure (i.e. voidage). The details of the

16 calculation of the coordination number with Suzuki's model are provided in the Appendix.

17 Suzuki's model takes into account the four cases of particle contact between the large (1)

18 and small (s) particles in the mixture, corresponding to cases (i)-(iv) described in the

19 Appendix. The average coordination number of the particles in a binary powder mixture, 
$1 N_{\mathrm{c}, \text { all }}^{*}$, is the sum of the average numbers of the four types of particle contacts shown in the

2 system, i.e.:

4

$$
\begin{aligned}
N_{\mathrm{c}, \text { all }}^{*} & =N_{\mathrm{c}(1-1)}^{*}+N_{\mathrm{c}(\mathrm{s}-\mathrm{s})}^{*}+N_{\mathrm{c}(1-\mathrm{s})}^{*}+N_{\mathrm{c}(\mathrm{s}-1)}^{*}, \\
& =N_{\mathrm{c}(1-1)}^{*}+N_{\mathrm{c}(\mathrm{s}-\mathrm{s})}^{*}+N_{\mathrm{c}(1, \mathrm{~s})}^{*}
\end{aligned}
$$

$6 \quad$ where $N_{\mathrm{c}(1, \mathrm{~s})}^{*}$ is the sum of $N_{\mathrm{c}(1-\mathrm{s})}^{*}$ and $N_{\mathrm{c}(\mathrm{s}-1)}^{*}$, representing the average number of contacts

7 between large and small particles, corresponding to ballotini and PS-DVB, respectively.

When an incipient flow occurs in a powder, the incipient shear zone is usually thin [19] as

9 shown in the two-dimensional schematic diagram in Fig. 6. In the case of single-component

10 powders, the thickness of the incipient shear zone is equivalent to the length of approximately

11 10-20 particles, regardless of the particle sizes [25-27]. Here, let us assume that the bulk

12 cohesion of binary powder mixtures can be expressed as a linear superposition of the numbers of three types of particle contacts existing in the incipient shear zone, i.e.:

$$
\tau_{\mathrm{c}}\left(x_{\mathrm{s}}\right)=A \cdot N_{\mathrm{c}, \mathrm{sh}(1-1)}\left(x_{\mathrm{s}}\right)+B \cdot N_{\mathrm{c}, \mathrm{sh}(\mathrm{s}-\mathrm{s})}\left(x_{\mathrm{s}}\right)+C \cdot N_{\mathrm{c}, \mathrm{sh}(1, \mathrm{~s})}\left(x_{\mathrm{s}}\right),
$$

where $N_{\mathrm{c}, \mathrm{sh}(1-1)}, N_{\mathrm{c}, \mathrm{sh}(\mathrm{s}-\mathrm{s})}$, and $N_{\mathrm{c}, \mathrm{sh}(1, \mathrm{c})}$ are the numbers of the contacts of large-large, small-small, and large-small particles being involved in the shear motion or existing in the incipient shear zone respectively, and $A, B$, and $C$ are coefficients with units of stress. By introducing a 
1 dimensionless function, $f=f\left(x_{\mathrm{s}}\right)$, which is proportional to the thickness of the incipient shear

2 zone in the powder mixtures, the number of three types of particle contacts, ballotini-ballotini,

3 (PS-DVB)-(PS-DVB), and ballotini-(PS-DVB), in the incipient shear zone can be expressed

4 by the following equations:

5

$6 \quad N_{\mathrm{c}, \mathrm{sh}(1-1)} \propto f \cdot n_{\mathrm{tot}} \cdot N_{\mathrm{c}(1-1)}^{*}$,

$7 \quad N_{\mathrm{c}, \mathrm{sh}(\mathrm{s}-\mathrm{s})} \propto f \cdot n_{\mathrm{tot}} \cdot N_{\mathrm{c}(\mathrm{s}-\mathrm{s})}^{*}$,

$8 \quad N_{\mathrm{c}, \mathrm{sh}(1, \mathrm{~s})} \propto f \cdot n_{\mathrm{tot}} \cdot N_{\mathrm{c}(1, \mathrm{~s})}^{*}$,

9

10 where $n_{\text {tot }}\left[\mathrm{m}^{-3}\right]$ is the number density of the total particles in the powder mixture. Therefore,

11 Eq. (5) can be rewritten as follows:

$13 \tau_{\mathrm{c}}\left(x_{\mathrm{s}}\right)=f\left(x_{\mathrm{s}}\right) \cdot n_{\mathrm{tot}}\left(x_{\mathrm{s}}\right) \cdot\left[A^{\prime} \cdot N_{\mathrm{c}(1-1)}^{*}\left(x_{\mathrm{s}}\right)+B^{\prime} \cdot N_{\mathrm{c}(\mathrm{s}-\mathrm{s})}^{*}\left(x_{\mathrm{s}}\right)+C^{\prime} \cdot N_{\mathrm{c}(1, \mathrm{~s})}^{*}\left(x_{\mathrm{s}}\right)\right]$,

15 where $A^{\prime}, B^{\prime}$, and $C^{\prime}$ are coefficients with units of stress times volume (i.e. $\mathrm{Pa} \mathrm{m}^{3}$ ), and are

16 measures of the relative degree of adhesion between ballotini-ballotini, (PS-DVB)-(PS-DVB),

17 and ballotini-(PS-DVB) contacts, respectively.

In Eq. (7), $f\left(x_{\mathrm{s}}\right) \cdot n_{\mathrm{tot}}\left(x_{\mathrm{s}}\right)$ is a relative measure of the number of total particles in the incipient shear zone. Since the thickness of the incipient shear zone is expected to be nearly 
1 proportional to (typically 10-20 times as large as) the diameter of particles in single

2 component system according to the discussion in Ref. [23-25], that in large-and-small binary

3 mixtures is also expected to be nearly proportional to the average diameter of the particle in

4 the system as discussed in Ref. [8] in general. The average diameter, $d_{\text {ave, }}$ is defined as

5 follows:

6

$7 \quad d_{\mathrm{ave}}=S_{\mathrm{n}, 1} \cdot d_{1}+S_{\mathrm{n}, \mathrm{s}} \cdot d_{\mathrm{s}}$,

9 where $S_{\mathrm{n}, 1}$ and $S_{\mathrm{n}, \mathrm{s}}\left(=1-S_{\mathrm{n}, 1}\right)$ are the number ratios of large and small particles, respectively.

10 Fig. 7a shows the results of such a calculation of $d_{\mathrm{ave}} \cdot n_{\mathrm{tot}}$ as a function of $x_{\mathrm{s}}$, in which $d_{\mathrm{ave}} \cdot n_{\mathrm{tot}}$

11 shows nearly a linear dependence on $x_{\mathrm{s}}$ in each case. Taking into account the fact that $f\left(x_{\mathrm{s}}\right)$ is

12 nearly proportional to $d_{\mathrm{ave}}$, the number of total particles in the incipient shear zone, i.e.

$13 f\left(x_{\mathrm{s}}\right) \cdot n_{\mathrm{tot}}\left(x_{\mathrm{s}}\right)$, is also assumed to increase almost linearly with increasing $x_{\mathrm{s}}$ as shown by the

14 dashed line in Fig. 7b. Also, it is reasonable to suppose that ratio of the incipient shear zone

15 thickness of the pure larger powder (i.e. glass ballotini) to that of pure smaller powder (i.e.

16 surface-modified PS-DVB) is equivalent to the ratio of their average diameters, i.e.:

$18 \quad \frac{f(1)}{f(0)} \approx \frac{d_{\mathrm{s}}}{d_{1}}$ 
1 In order to simplify the calculation, let $f(1)$ be equal to 1 and therefore $f(0)=d_{1} / d_{\mathrm{s}}$. By

2 calculating the gradient $a$ in Fig. $7 \mathrm{~b}$ by $d_{1}, d_{\mathrm{s}}, n_{\mathrm{tot}, \mathrm{l}}$, and $n_{\mathrm{tot}, \mathrm{s}}$, the function $f\left(x_{\mathrm{s}}\right)$ is defined by

3 the following equation:

4

$5 \quad f\left(x_{\mathrm{s}}\right)=\frac{\left[n_{\mathrm{tot}, \mathrm{s}}-\left(d_{1} / d_{\mathrm{s}}\right) \cdot n_{\mathrm{tot}, 1}\right] \cdot x_{\mathrm{s}}+\left(d_{1} / d_{\mathrm{s}}\right) \cdot n_{\mathrm{tot}, 1}}{n_{\mathrm{tot}}\left(x_{\mathrm{s}}\right)}$.

6

7 Now, the bulk cohesion of the powder mixtures can be calculated by assigning Eq. (10) to the

8 function $f\left(x_{\mathrm{s}}\right)$ in Eq. (7).

9 In Eq. (7), the value of $A^{\prime}$ is specified by assigning $x_{\mathrm{s}}=0$ and applying $f=d_{1} / d_{\mathrm{s}}$ as

$10 A^{\prime}=\left[\tau_{\mathrm{c}, 1} /\left(n_{\mathrm{tot}, 1} \cdot N_{\mathrm{c}, 1}\right)\right] \cdot\left(d_{\mathrm{s}} / d_{1}\right)$, where $\tau_{\mathrm{c}, 1}, n_{\mathrm{tot}, 1}$, and $N_{\mathrm{c}, 1}$, are the bulk cohesion, number

11 density, and coordination number of the glass ballotini, respectively. In the same manner,

$12 B^{\prime}=\tau_{\mathrm{c}, \mathrm{s}} /\left(n_{\mathrm{tot}, \mathrm{s}} \cdot N_{\mathrm{c}, \mathrm{s}}\right)$ is obtained by substituting $x_{\mathrm{s}}=1$ into Eq. (7) and applying $f=1$, where

$13 \tau_{\mathrm{c}, \mathrm{s}}, n_{\mathrm{tot}, \mathrm{s}}$, and $N_{\mathrm{c}, \mathrm{s}}$, are the bulk cohesion, number density, and coordination number of the

14 surface-modified PS-DVB powder, respectively. Therefore, the coefficient $C^{\prime}$ is the only

15 variable parameter to be fitted.

16 Fig. 8 shows the results of such a calculation. For the mixtures of glass ballotini and

17 PS-DVB(100), the model fits well to the experimental data over almost the entire range of $x_{\mathrm{s}}$

18 by choosing an appropriate value for $C^{\prime}$ (typical values are tabulated in Table 5). However, in

19 the case of the powder mixtures of glass ballotini and PS-DVB(1), large discrepancies were 
1 found between the results of calculation and experimental data in the small $x_{\mathrm{s}}$ region,

2 although they agree well with each other when $x_{\mathrm{s}}$ exceeds 0.1 . In fact, this discrepancy

3 becomes more obvious as the bulk cohesion of the surface-modified PS-DVB powder

4 becomes larger.

5 The bulk cohesion of the surface-modified PS-DVB powders depends on how much the

6 surface additives on the PS-DVB particles disrupt their direct contacts; the more efficiently

7 the additives disrupt the direct contact between the PS-DVB particles, the smaller the bulk

8 cohesion, as reported in [20]. When it comes to the adhesion with glass ballotini, the

9 surface-modified PS-DVB particles with smaller bulk cohesion are generally supposed to

10 have a weaker adhesion since the additives on such PS-DVB particles must disrupt the direct

11 contact between glass ballotini and PS-DVB particles more efficiently.

12 However, as the adhesion between the glass ballotini and surface-modified PS-DVB

13 particles increases, the PS-DVB particles between the glass ballotini particles may work as if

14 they are adhesives, causing a rapid increase of the number of the particles involved in the

15 shear motion, $f\left(x_{\mathrm{s}}\right) \cdot n_{\mathrm{tot}}\left(x_{\mathrm{s}}\right)$, for very small $x_{\mathrm{s}}$. Modifying the proposed relationship between

$16 f\left(x_{\mathrm{s}}\right) \cdot n_{\mathrm{tot}}\left(x_{\mathrm{s}}\right)$ and $x_{\mathrm{s}}$ to a linear function whose gradient is $(1-\delta) \cdot a$ after $f\left(x_{\mathrm{s}}\right) \cdot n_{\mathrm{tot}}\left(x_{\mathrm{s}}\right)$ increases

17 rapidly at very small $x_{\mathrm{s}}$, as illustrated by the solid line in Fig. $7 \mathrm{~b}$, the function $f$ can be

18 denoted by the following equation: 
$1 f\left(x_{\mathrm{s}}\right)=\left\{\begin{array}{cc}\frac{(1-\delta) \cdot\left[n_{\mathrm{tot}, \mathrm{s}}-\left(d_{1} / d_{\mathrm{s}}\right) \cdot n_{\mathrm{tot}, 1}\right] \cdot x_{\mathrm{s}}+n_{\mathrm{tot}, \mathrm{s}}-(1-\delta) \cdot\left[n_{\mathrm{tot}, \mathrm{s}}-\left(d_{1} / d_{\mathrm{s}}\right) \cdot n_{\mathrm{tot}, 1}\right]}{n_{\mathrm{tot}}\left(x_{\mathrm{s}}\right)} & \left(x_{\mathrm{s}}>0\right) \\ \frac{d_{1}}{d_{\mathrm{s}}} & \left(x_{\mathrm{s}}=0\right)\end{array}\right.$.

3 The bulk cohesion of the powder mixtures was then recalculated by substituting Eq. (11) into

4 Eq. (7), instead of Eq. (10).

5 The coefficients $A^{\prime}$ and $B^{\prime}$ were again determined as $A^{\prime}=\left[\tau_{\mathrm{c}, 1} /\left(n_{\mathrm{tot}, 1} \cdot N_{\mathrm{c}, 1}\right)\right] \cdot\left(d_{\mathrm{s}} / d_{1}\right)$ and

$6 \quad B^{\prime}=\tau_{\mathrm{c}, \mathrm{s}} /\left(n_{\mathrm{tot}, \mathrm{s}} \cdot N_{\mathrm{c}, \mathrm{s}}\right)$, which can be calculated by substituting $x_{\mathrm{s}}=0$ and 1 , respectively. The

7 two parameters, $C^{\prime}$ and $\delta$, were chosen so that Eq. (7) fitted best to the experimental results.

8 The model calculation shows good agreement with the experimental data when choosing

9 appropriate values for $C^{\prime}$ and $\delta$ for the mixtures with each type of surface-modified PS-DVB

10 powder, as shown in Fig. 9. The values of $B^{\prime}$ obtained by calculation, as well as those of $C^{\prime}$

11 and $\delta$ obtained by fitting, are as listed in Table 5. The value of $A^{\prime}$ was fixed at $1.0 \times 10^{-12} \mathrm{~Pa}$

$12 \mathrm{~m}^{3}$.

\section{Analysis of data based on model}


As mentioned in Section 5, the values of $A^{\prime}, B^{\prime}$, and $C^{\prime}$ are the relative measures of the

2 degree of adhesion between ballotini-ballotini, (PS-DVB)-(PS-DVB), and

3 ballotini-(PS-DVB) contacts, respectively. Fig. 10 compares the values of $B^{\prime}$ and $C^{\prime}$ for each

4 type of binary powder mixture, which validates the assumption mentioned above; namely,

5 that the more surface additives cover the surface of a PS-DVB particle, the less chance the

6 surface-modified PS-DVB particle has of making direct contact, not only with other PS-DVB

7 particles, but also with glass ballotini. The parameter $G$ in Eq. (3), representing how easily

8 the binary mixture can be compacted, also has a strong correlation with the parameter $C^{\prime}$, as

9 shown in Fig. 11, which implies that $G$ also has a correlation with $B^{\prime}$. This demonstrates that

10 the packing structure of the binary powder mixtures and the adhesion between the two types

11 of constituent particles are strongly related with each other, similar to the fact that the

12 packing structure of the single component PS-DVB powders is correlated with their bulk

13 cohesion, as seen in Fig. 3.

14

6.2. Thickness of the incipient shear zone

17 Since the function $f\left(x_{\mathrm{s}}\right)$ is defined as a relative measure of the thickness of the incipient

18 shear zone in the binary powder mixtures, it is possible to analyse the thickness of the

19 incipient shear zone quantitatively in terms of its dependence on $x_{\mathrm{s}}$, as well as effects of the 
1 surface-modified PS-DVB. Fig. 12 compares the function $f\left(x_{\mathrm{s}}\right)$ for all of the binary powder

2 mixtures, where the lines are the calculated values based on the linear change of $f\left(x_{\mathrm{s}}\right) \cdot n_{\mathrm{tot}}\left(x_{\mathrm{s}}\right)$

3 and the squares are the recalculated values so that the result of model calculation of Eq. (9)

4 agrees with the experimentally obtained bulk cohesion using the values of parameters $A^{\prime}, B^{\prime}$,

$5 \quad C^{\prime}$, and $\delta$ listed in Table 5 .

6 In each case, the function $f\left(x_{\mathrm{s}}\right)$ decreases with increasing $x_{\mathrm{s}}$, which seems to reflect the

7 decrease of the average diameter of the powder mixtures. The function $f\left(x_{\mathrm{s}}\right)$ increases as the

8 parameter $\delta$ becomes larger, as defined in Eq. (11), so the parameter $\delta$ represents the degree

9 of the increase of the incipient shear zone thickness, or the number of particles in the

10 incipient shear zone. In fact, the values of $\delta$ show a good correlation with those of the

11 coefficient $C^{\prime}$, as shown in Fig. 13. This fact indicates that the stronger the adhesion between

12 the glass ballotini and PS-DVB particles, the thicker the incipient shear zone becomes, for the

13 same values of $x_{\mathrm{s}}$.

14 Some values of the function $f$ at very small $x_{\mathrm{s}}$ (i.e. $\left.x_{\mathrm{s}}=\sim 0.002\right)$ obtained from the

15 experimental data (presented in Fig. 12 by dots) show relatively large deviation from the

16 calculated values (lines), in such cases as the mixtures of glass ballotini and PS-DVB(1) or

17 PS-DVB(100). These seem to reflect the fact that the function $f\left(x_{\mathrm{s}}\right)$ should converge on $d_{\mathrm{l}} / d_{\mathrm{s}}$

$18 \quad(=9.17)$ as $x_{\mathrm{s}}$ approaches to 0. 
Equation (7) has three terms, $f \cdot n_{\mathrm{tot}} \cdot A^{\prime} \cdot N_{\mathrm{c}(1-1)}^{*}, \quad f \cdot n_{\mathrm{tot}} \cdot B^{\prime} \cdot N_{\mathrm{c}(\mathrm{s}-\mathrm{s})}^{*}, \quad$ and

$4 f \cdot n_{\mathrm{tot}} \cdot C^{\prime} \cdot N_{\mathrm{c}(1, \mathrm{~s})}^{*}$, each of which represents the parts of the cohesion of ballotini-ballotini,

5 (PS-DVB)-(PS-DVB), and ballotini-(PS-DVB) contacts, respectively, in the total bulk

6 cohesion of the powder mixtures. As an example, these three terms calculated using the

7 values of the parameters $A^{\prime}, B^{\prime}, C^{\prime}$, and $\delta$ listed in Table 5 and the function $f$ which fits best

8 with the experimental data for the mixtures of the glass ballotini and PS-DVB(10) are

9 presented in Fig. 14. As illustrated, the bulk cohesion of the binary powder mixtures is found

10 to be divided into three regions by comparing the values of these three terms. The

11 ballotini-ballotini contacts are dominant in Region I $\left(x_{\mathrm{s}}<0.025\right)$, where their cohesion

12 rapidly decreases and that of ballotini-(PS-DVB) contacts gradually increases, whilst the

13 (PS-DVB)-(PS-DVB) contacts have almost no effect. In Region II $\left(0.025<x_{\mathrm{s}}<0.28\right)$, the

14 ballotini-(PS-DVB) contacts are the primary contribution to the total bulk cohesion, where

15 the cohesion of ballotini-ballotini contacts approaches zero and that of (PS-DVB)-(PS-DVB)

16 contacts starts increasing with increasing $x_{\mathrm{s}}$. The cohesion of (PS-DVB)-(PS-DVB) contacts

17 keeps increasing and exceeds that of ballotini-(PS-DVB), and then becomes dominant in

18 Region III $\left(x_{\mathrm{s}}>0.28\right)$.

19 The same analysis is applicable to all other powder mixtures. By comparing each term 
1 separately, it is possible to discuss the effect of the difference of surface-modified PS-DVB

2 powders on the total bulk cohesion in detail. Fig. 15 presents the three terms of the bulk

3 cohesion of each binary powder mixture. The bulk cohesion of the mixtures is different in the

4 entire range of $x_{\mathrm{s}}$ as shown in Fig. 5(a), which seems to be attributable to the difference in the

5 bulk cohesion of the PS-DVB powders, as mentioned in Section 4. In fact, the cohesion of

6 (PS-DVB)-(PS-DVB) contacts starts increasing once $x_{\mathrm{s}}$ reaches approximately 0.05 , and their

7 difference then becomes apparent with increasing $x_{\mathrm{s}}$, as shown in Fig. 15(b). On the other

8 hand, the cohesion of ballotini-ballotini contacts is different below $x_{\mathrm{s}}=\sim 0.05$ (Fig. 15(a)).

9 This reflects the value of $\delta$, which results in the variation of the number of glass ballotini

10 particles involved in the shear motion since the value of $A^{\prime}$ is common to all the powder

11 mixtures. The cohesion of ballotini-(PS-DVB) contacts is different over almost the entire

12 range of $x_{\mathrm{s}}$, particularly in $x_{\mathrm{s}}<\sim 0.4$ (Fig. 15 (c)), mainly due to the value of $C^{\prime}$, which also

13 affects the change of the total bulk cohesion.

6.4. Relationship with internal friction

17 By comparing Fig. 5(b) with Fig. 14, it can be found that the linearised friction angle

18 (LFA) at first decreases with the decreasing effect of ballotini-ballotini contacts as well as an increase of the effect of ballotini-(PS-DVB) contacts, and then turns into increase following 
1 the increase of the effect of (PS-DVB)-(PS-DVB) contacts. Therefore, the change of LFA

2 seems to reflect how the contribution of each type of particle contacts changes with increase

3 of $x_{\mathrm{s}}$ in each powder mixture.

4 Fig. 16 compares the ratio of the three types of cohesion to the total bulk cohesion,

5 representing the contribution of each type of particle contacts to the total bulk flow property.

6 Fig. 16 illustrates that, by using a less cohesive surface-modified PS-DVB powder, both the

7 decrease of the contribution of ballotini-ballotini contacts and increase of that of

8 (PS-DVB)-(PS-DVB) contacts shift in the direction of larger $x_{\mathrm{s}}$, and so does the peak of the

9 contribution of ballotini-(PS-DVB) contacts. As explained in Section 4, the ratio of the

10 surface-modified PS-DVB particles in which the LFA becomes minimum, $x_{\mathrm{s}}\left(\varphi_{\operatorname{lin}, \min }\right)$,

11 increases as the bulk cohesion of the surface-modified PS-DVB becomes smaller (Fig. 5(b)),

12 and this corresponds to the shift of the contributions of each type of particle contacts seen in

13 Fig. 16. This fact strongly supports that the suggested model correctly describes the effect of

14 the adhesion between the constituent particles into the bulk flow properties.

15 Fig. 17 compares $x_{\mathrm{s}}\left(\varphi_{\text {lin,min }}\right)$ and the boundary between Regions II and III defined in Fig.

$1614, x_{\mathrm{s}}(1 \mathrm{~s}-\mathrm{ss})$, for all types of the binary powder mixtures, showing the fact that the values of

$17 x_{\mathrm{s}}\left(\varphi_{\text {lin,min }}\right)$ and $x_{\mathrm{s}}(1 \mathrm{~s}-\mathrm{ss})$ have a strong correlation with each other; the values of $x_{\mathrm{s}}\left(\varphi_{\text {lin,min }}\right)$ are

18 always slightly (i.e. 0.04-0.06) smaller than that of $x_{\mathrm{s}}(1 \mathrm{~s}-\mathrm{ss})$. 


\section{7. Conclusions}

3

4 In this study, we have experimentally investigated the bulk flow properties of

5 large-and-small fine binary powder mixtures as a function of the ratio of the component

6 powders, and proposed a semi-empirical model describing their relationship incorporating the

7 contacts between the constituent particles.

8 First, the incipient flow properties of the binary powder mixtures were evaluated using

9 large-and-small model powder mixtures (of diameter 71.9 and $7.84 \mu \mathrm{m}$, respectively).

10 Systematic measurements were conducted using polymer microspheres as smaller particles

11 whose bulk cohesion was controlled by surface additives. The two flow parameters: bulk

12 cohesion, $\tau_{\mathrm{c}}$, and linearised friction angle (LFA), $\varphi_{\text {lin, }}$, were obtained from the yield loci

13 obtained by ring shear testing.

14 The flow properties of the powder mixtures were then compared with their packing

15 structure. The macroscopic structure of the powder mixtures was evaluated in the form of

16 voidage, and then the coordination number of the particles in the mixtures was estimated

17 using Suzuki's model. With these data, we have developed a semi-empirical model equation

18 in which the total bulk cohesion of the powder mixtures is expressed as a linear superposition

19 of the numbers of the three types of particle contacts involved in the shear motion. An 
1 assumption that the number of particles in the incipient shear zone has a linear correlation

2 with the volume ratio of the smaller constituent particles (i.e. surface-modified PS-DVB), $x_{\mathrm{s}}$,

3 was made.

4 The model equation reproduced the experimental data of the bulk cohesion well by

5 choosing appropriate values to the two parameters: $C^{\prime}$, representing the adhesive property

6 between large and small particles, and $\delta$, accounting for the rapid increase of the number of

7 particles in the incipient shear zone at low $x_{\mathrm{s}}$ region. The values of the parameter $\delta$ were

8 highly correlated with those of $C^{\prime}$, which indicated that the stronger the adhesion between the

9 large and small particles, the thicker the incipient shear zone becomes, at very small $x_{\mathrm{s}}$

10 region.

11 By decomposing the bulk cohesion of each powder mixture into three fractions according

12 to the contribution of the types of particle contacts: large-large, large-small, and small-small

13 particle contacts, the bulk cohesion was divided into three regions with increasing $x_{\mathrm{s}}$ in which

14 each of these three types of particle contacts is dominant. Also, the following findings were

15 obtained by further analyses:

17 - The total bulk cohesion of the mixtures with more cohesive small constituent became

18 higher throughout the entire range of $x_{\mathrm{s}}$. This is attributed to the increase of the incipient

19 shear zone thickness as well as stronger adhesion of large-small particle contacts for $x_{\mathrm{s}}<$ 
$1 \sim 0.05$, whilst this reflects the cohesion of small-small particle contacts for $x_{\mathrm{s}}>\sim 0.05$.

2

3 - By using less cohesive smaller particles, the value of $x_{\mathrm{s}}$ at which the contribution of

4 large-small particle contacts in the total bulk cohesion reached a maximum shifted in the

$5 \quad$ direction of larger $x_{\mathrm{s}}$.

6

7 - The LFA followed a funnel-shaped curve in terms of $x_{\mathrm{s}}$, and the value of $x_{\mathrm{s}}$ at which the

8 LFA reached a minimum, $x_{\mathrm{s}}\left(\varphi_{\text {lin,min }}\right)$, shifted in the direction of larger $x_{\mathrm{s}}$ when using less

9 cohesive smaller particles. This corresponded to the shift of the maximum contribution of

10 large-small particle contacts in bulk cohesion.

As potential further work, the range of validity of the proposed model can be explored.

13 The degrees of adhesion of the three types of particle contacts were compared quantitatively

14 by their relative measures obtained in the model calculation: $A^{\prime}, B^{\prime}$, and $C^{\prime}$, which were

15 proven here to have a well-defined physical interpretation. However, direct measurements of

16 the adhesive force between particles, by means of techniques such as atomic force

17 microscopy (AFM) as some studies have attempted [28-31], would provide a useful

18 comparison to the results presented here. Also, the physical description of powder flow in this

19 model can be further enhanced by numerical model simulations such as discrete element 
1 method (DEM). The dependence of the flow properties of binary powder mixtures on the

2 consolidation stress would also be of further interest.

3 Lastly, an investigation of the range of powder size as well as size ratio in which the

4 proposed model is applicable can be carried out. The model works quite well in the case that

5 the constituent particles are micrometre-sized, and the size ratio of large and small

6 components is at least $\sim 10: 1$. The cases where the larger particles' average size exceeds

7 approximately $100 \mu \mathrm{m}$, and therefore the influence of gravity will overcome the interparticle

8 adhesive force, or where the smaller particles' size is less than approximately $1 \mu \mathrm{m}$, and the

9 particles will tend to agglomerate strongly, would be of interest to study.

10 In summary, the achievements of this study are expected to advance the understanding of

11 the fundamental flow properties of fine powder mixtures. Also, they will give a clear

12 direction of the bulk flow properties of component powders in the design of powder mixtures

13 and realise the better control of the flow behaviour in the products such as EP developers.

17 Appendix: Coordination number

19 Here, a geometric model for calculating the coordination number of the particles in 
1 large-and-small binary powder mixtures proposed by Suzuki and co-workers [12-14], which

2 is applied to the analysis of the relationship between flow properties and particle contacts of

3 the binary powder mixtures in this study, is illustrated in detail.

4 The model takes into account the four different cases of particle contact, i.e.:

6 Case (i): Large particles in contact with a reference large particle;

7 Case (ii): Small particles in contact with a reference large particle;

8 Case (iii): Large particles in contact with a reference small particle;

9 Case (iv): Small particles in contact with a reference small particle,

11 as illustrated in detail in Refs. [2,13]. The number of particles making contact with a

12 reference particle in cases (i) and (iv) can be obtained by considering a powder composed of

13 uniform-sized spherical particles. In the case of powder with uniform-sized spheres with

14 diameter $d$, the relationship between the coordination number $N_{\mathrm{c}, \mathrm{u}}$ and voidage $\varepsilon$ of the

15 powder is expressed by the following empirical equation:

$17 \quad N_{\mathrm{c}, \mathrm{u}}=2.812 \frac{(1-\varepsilon)^{-1 / 3}}{(b / d)\left[1+(b / d)^{2}\right]}$,

19 where $b$ is a coefficient determined by the voidage $\varepsilon$, calculated from: 
$2(1-\varepsilon)^{-1 / 3}=\frac{1+(b / d)^{2}}{1+(b / d) \exp \left[(d / b)^{2}\right] \cdot \operatorname{Erfc}(d / b)}$,

4 where $\operatorname{Erfc}(x)$ represents the complementary Gaussian error function.

5 In case (ii), the number of small particles making contact with a reference large particle,

$6 \quad N_{\mathrm{c}(1-\mathrm{s})}$, is expressed by the following equation:

7

$8 \quad N_{\mathrm{c}(\mathrm{l}-\mathrm{s})}=\frac{N_{\mathrm{c}, \mathrm{u}}(2-\sqrt{3})\left[\left(d_{1} / d_{\mathrm{s}}\right)+1\right]}{2\left[1+\left(d_{1} / d_{\mathrm{s}}\right)-\left\{\left(d_{1} / d_{\mathrm{s}}\right)\left[\left(d_{1} / d_{\mathrm{s}}\right)+2\right]\right\}^{1 / 2}\right]}$,

9

10 where $d_{1}$ and $d_{\mathrm{s}}$ are the diameters of large and small powders, respectively. In the same

11 manner, the number of large particles contacting with a reference small particle, case (iii),

$12 N_{\mathrm{c}(\mathrm{s}-\mathrm{l})}$, can be calculated by exchanging the quantities $d_{1}$ and $d_{\mathrm{s}}$ in Eq. (A3).

13 The coordination number of the large powder, $N_{\mathrm{c}, \mathrm{l}}$, can then be calculated by making the

14 assumption that the number fraction of the contact particles on the reference particle is related

15 to the surface area occupied by the individual contact particle as follows, taking into account

16 cases (i) and (ii):

17

$18 N_{\mathrm{c}, 1}=S_{\mathrm{a}, 1} N_{\mathrm{c}(1-1)}+S_{\mathrm{a}, 5} N_{\mathrm{c}(1-\mathrm{s})}$, 
2 where $S_{\mathrm{a}, \mathrm{l}}\left(\right.$ or $\left.S_{\mathrm{a}, \mathrm{s}}\right)$ is the fractional area ratio of the large (small) powder, defined as the ratio

3 of the total surface area of the large (small) particles in the surface area of all of the particles

4 in the system, with $S_{\mathrm{a}, \mathrm{s}}=1-S_{\mathrm{a}, \mathrm{l}}$. The first and second terms in Eq. (A4) represent the number

5 of large-large and large-small particle contacts per a large particle in the powder mixture,

6 respectively. The coordination number of the small powder, $N_{\mathrm{c}, \mathrm{s}}$, can be obtained in the same

7 manner, i.e.:

8

$9 \quad N_{\mathrm{c}, \mathrm{s}}=S_{\mathrm{a}, 1} N_{\mathrm{c}(\mathrm{s}-1)}+S_{\mathrm{a}, \mathrm{s}} N_{\mathrm{c}(\mathrm{s}-\mathrm{s})}$,

11 where the first and second terms in Eq (A5) represent the number of small-large and

12 small-small particle contacts per small particle in the mixture, respectively.

13 The average coordination number of the total binary powder system, $N_{\mathrm{c}, \text { all }}^{*}$, is the

14 summation of the products of the coordination numbers of each particle, $N_{\mathrm{c}, 1}$ and $N_{\mathrm{c}, \mathrm{s}}$, defined

15 in Eqs. (A4) and (A5), and the number ratio of the large (small) powder particles in the total

16 powder system, $S_{\mathrm{n}, 1}\left(S_{\mathrm{n}, \mathrm{s}}\right)$, i.e.:

17

18

$$
\begin{aligned}
N_{\mathrm{c}, \mathrm{all}}^{*} & =S_{\mathrm{n}, 1} N_{\mathrm{c}, 1}+S_{\mathrm{n}, \mathrm{s}} N_{\mathrm{c}, \mathrm{s}} \\
& =S_{\mathrm{n}, 1} S_{\mathrm{a}, 1} N_{\mathrm{c}(1-1)}+S_{\mathrm{n}, \mathrm{s}} S_{\mathrm{a}, \mathrm{s}} N_{\mathrm{c}(\mathrm{s}-\mathrm{s})}+S_{\mathrm{n}, 1} S_{\mathrm{a}, \mathrm{s}} N_{\mathrm{c}(1-\mathrm{s})}+S_{\mathrm{n}, \mathrm{s}} S_{\mathrm{a}, 1} N_{\mathrm{c}(\mathrm{s}-1)}
\end{aligned} .
$$


1 The first to fourth terms in Eq. (A6) correspond to the average numbers of the particle

2 contacts in cases (i)-(iv): $N_{\mathrm{c}(1-1)}^{*}, \quad N_{\mathrm{c}(s-s)}^{*}, N_{\mathrm{c}(1-\mathrm{s})}^{*}$ and $N_{\mathrm{c}(s-1)}^{*}$, respectively.

3

4

5

6 References

7

8 [1] L. B. Schein, Electrophotography and Development Physics, 2nd revis. ed., Springer, $9 \quad$ New York, 1992, p. 26.

10 [2] T. Kojima, J. A. Elliott, Incipient flow properties of two-component fine powder systems 11 and their relationships with bulk density and particle contacts, Powder Technology 228 $12 \quad$ (2012) 359-370.

13 [3] J. Hidaka, Y. Sasaki, A. Shimosaka, Y. Shirakawa, Simulation of flow behavior of 14 tow-component developer in electro-photographic system, Advanced Powder 15 Technology 13 (2002) 317-332.

16 [4] O. Molerus, M. Nywlt, The influence of the fine particle content on the flow behaviour of 17 bulk materials, Powder Technolgy 37 (1984) 145-154.

18 [5] L. E. Vallejo, Interpretation of the limits in shear strength in binary granular mixtures.

19 Canadian Geotechnical Journal 38 (2001) 1097-1104. 
1 [6] Z. Chik, L. E. Vallejo, Characterization of the angle of repose of binary granular materials,

2 Canadian Geotechnical Journal 42 (2005) 683-692.

3 [7] T. Ueda, T. Matsushima, Y. Yamada, Effect of particle size ratio and volume fraction on

4 shear strength of binary granular mixture, Granular Matter 13 (2011) 731-742.

5 [8] T. Ueda, T. Matsushima, Y. Yamada, Ball-bearing effect on shear behavior of binary

6 granular mixture, Journal of Japan Society of Civil Engineers, Ser. A2 68 (2012) 1-9.

7 [9] D. Pinson, P. R. Zou, A. B. Yu, P. Zulli, M. J. McCarthy, Coordination number of binary

8 mixtures of spheres, Journal of Physics D: Applied Physics 31 (1998) 457-462.

9 [10] P. R. Zou, M. I. Gan, A. B. Yu, Prediction of the porosity of multi-component mixtures

10 of cohesive and non-cohesive particles, Chemical Engineering Science 66 (2011)

$11 \quad 4711-4721$.

12 [11] N. Ouchiyama, T. Tanaka, Estimation of the average number of contacts between 13 randomly mixed solid particles, Industrial and Engineering Chemistry Fundamentals 19

$14 \quad$ (1980) 338-340.

15 [12] M. Suzuki, K. Makino, M. Yamada, K. Iinoya, A study of coordination number in a 16 random packed system of monosized sphere particles, Kagaku Kogaku Ronbunshu 6 17 (1980) 59-64.

18 [13] M. Suzuki, T. Oshima, Estimation of the co-ordination number in a multi-component mixture of spheres, Powder Technology 35 (1983) 159-166. 
1 [14] M. Suzuki, T. Oshima, Estimation of the co-ordination number in a two-component

2 mixture of cohesive spheres, Powder Technology 36 (1983) 181-188.

3 [15] V. F. Pershin, Calculation of the relative density and coordination number of a 4 multiparticle-sized powder, Powder Metallurgy and Metal Ceramics 29 (1990) 178-182.

5 [16] G. A. Georgalli, M. A. Reuter, Modelling the co-ordination number of a packed bed of

6 spheres with distributed sizes using a CT scanner, Minerals Engineering 19 (2006)

$7 \quad 246-255$.

8 [17] L. Y. Yi, K. J. Dong, P. R. Zou, A. B. Yu, Coordination number of the packing of ternary

9 mixtures of spheres: DEM simulations versus measurements, Industrial and Engineering

$10 \quad$ Chemical Research 30 (2011) 8773-8785.

11 [18] R. M. Nedderman, Statics and Kinematics of Granular Materials, Cambridge University

12 Press, Cambridge, 1992.

13 [19] D. Schulze, Powders and Bulk Solids: Behavior, Characterization, Storage and Flow, 14 Springer-Verlag, Heidelberg, 2008.

15 [20] T. Kojima, J. A. Elliott, Effect of silica nanoparticles on the bulk flow properties of fine 16 cohesive powders, Chemical Engineering Science 101 (2013) 315-328.

17 [21] A. B. Yu, J. Bridgwater, A. Burbidge, On the modelling of the packing of fine particles, Powder Technology 92 (1997) 185-194.

19 [22] S. Liu, Z. Ha, Prediction of random packing limit for multimodal particle mixtures, 
2 [23] A. E. R. Westman, The packing of particles: empirical equations for intermediate diameter ratios, Journal of American Ceramic Society 19 (1936) 127-129.

[24] A. B. Yu, N. Standish, A. McLean, Porosity calculation of binary mixtures of nonspherical particles, Journal of American Ceramic Society 76, (1993) 2813-2816.

[25] H. -B. Mühlhaus, I. Vardoulakis, The thickness of shear bands in granular materials, Geotechnique 37 (1987) 271-283.

[26] C. Han, A. Drescher, Shear bands in biaxial tests on dry coarse sand, Soils and Fundations 33 (1993) 118-132.

10 [27] T. B. S. Pradhan, in Deformation and Progressive Failure in Geomechanics, edited by A.

11 Asaoka, T. Adachi, F. Oka, Pergamon, Oxford, 1997, p. 241.

12 [28] R. Jones, From single particle AFM studies of adhesion and friction to bulk flow: 13 forging the links, Granular Matter 4 (2003) 191-204.

14 [29] A. Castellanos, The relationship between attractive interparticle forces and bulk behaviour in dry and uncharged fine powders, Advances in Physics 54 (2005) 263-376.

16 [30] M. A. S. Quintanilla, A. Castellanos, J. M. Valverde, Correlation between bulk stresses and interparticle contact forces in fine powders, Physical Review E 64 (2001) 031301-1-9. 
$1 \quad$ No. 2894, 1928; Bulletin of US Bureau of Mines 307 (1929) 74.

2

3

4 